\title{
Fault-Tolerant Resolvability of Certain Crystal Structures
}

\author{
Sathish Krishnan 1,2, Bharati Rajan ${ }^{3,4}$ \\ ${ }^{1}$ Research and Development Centre, Bharathiar University, Coimbatore, India \\ ${ }^{2}$ Department of Mathematics, DMI College of Engineering, Chennai, India \\ ${ }^{3}$ Department of Mathematics, Loyola College, Chennai, India \\ ${ }^{4}$ School of Mathematical and Physical Sciences, Faculty of Science and IT, The University of Newcastle, \\ Callaghan, Australia \\ Email: satiskris@gmail.com,bharatirajan@gmail.com,bharati.rajan@newcastle.edu.au
}

Received 16 February 2016; accepted 22 April 2016; published 25 April 2016

Copyright (C) 2016 by authors and Scientific Research Publishing Inc.

This work is licensed under the Creative Commons Attribution International License (CC BY).

http://creativecommons.org/licenses/by/4.0/

(c) (i) Open Access

\begin{abstract}
An ordered set $W$ of vertices of a graph $G$ is called a resolving set, if all the vertices of $G$ are uniquely determined by the vector of distances to the vertices in $W$. The metric dimension of $G$ is the minimum cardinality of a resolving set of $G$. A resolving set $W$ for $G$ is fault-tolerant if $W \backslash\{v\}$ is also a resolving set, for each $v$ in $W$, and the fault-tolerant metric dimension of $G$ is the minimum cardinality of such a set. In this paper we determine the metric dimension and fault-tolerant metric dimension problems for the graphs of certain crystal structures.
\end{abstract}

\section{Keywords}

Resolving Set, Metric Dimension, Fault-Tolerant metric Dimension, Crystal Structures, Bismuth Tri-Iodide, Lead Chloride, Quartz

\section{Introduction}

Let $G$ be a connected graph with vertex set $V$ and edge set $E$. The distance $d(u, v)$ between two vertices $u, v \in V$ is the length of a shortest path between them. Let $W=\left\{w_{1}, w_{2}, \cdots, w_{k}\right\}$ be an ordered set of vertices of $G$ and let $v$ be a vertex of $G$. The representation $r(v \mid W)$ of $v$ with respect to $W$ is the $k$-tuple $\left(d\left(v, w_{1}\right), d\left(v, w_{2}\right), \cdots, d\left(v, w_{k}\right)\right)$. Then $W$ is a resolving set for $G$ if and only if no two vertices of $G$ have the same representation with respect to $W$. A resolving set of minimum cardinality is called a basis for $G$ and this cardinality is the metric dimension of $G$, denoted by $\beta(G)$. Note that $w_{i}$ is the only vertex of $W$ for which the ith co-ordinate of its representation with respect to $W$ is 0 . Therefore, when checking if $W$ is a resolving set for $G$, it suffices to consider pair of vertices $x$, 
$y \in V \backslash W$. A resolving set $W$ for $G$ is fault-tolerant if $W \backslash\{v\}$ is also a resolving set, for each $v$ in $W$. A fault-tolerant resolving set of minimum cardinality is called a fault-tolerant metric basis for $G$ and this cardinality is the fault-tolerant metric dimension of $G$, denoted by $\beta^{\prime}(G)$.

Slater [1] introduced the concept of a resolving set for a connected graph which was also independently discovered by Harary and Melter [2]. Melter et al. [3] studied the metric dimension problem for grid graphs induced by lattice points in the plane when the distances are measured in the $L_{1}$ and $L_{\infty}$ metrics. Khuller et al. [4] have proved that the metric dimension of a $d$-dimensional grid is $d$, generalizing the result of Melter et al. [3]. They have obtained a linear time algorithm to find the metric dimension of trees. They have considered graphs that require only a few landmarks and have shown that paths are the only graphs with $\beta=1$. Further they have shown that a graph $G$ with $\beta(G)=2$ can have neither $K_{5}$ nor $K_{3 ; 3}$ as a subgraph. By extending this they have stated that a graph $\mathrm{G}$ with $\beta(G)=k$ cannot have $K_{2^{k+1}}$ as a subgraph. Using these facts they have conjectured that graphs having $\beta(G)=2$ might be planar, but have exhibited a non-planar graph with $\beta(G)=2$. It is also shown that the metric dimension of a graph with $n$ nodes can be approximated in polynomial time within a factor of $O(\log n)$.

Hernando et al. [5] make a general study of the metric dimension of Cartesian products of graphs. Part of their motivation for studying the metric dimension of Cartesian products is that in two of the applications, namely Mastermind and coin weighing, the graphs that arise are Cartesian products. It was noted in [6] that determining the metric dimension of a graph is an NP-hard problem. Manuel et al. [7] have shown that the problem remains NP-complete for bipartite graphs. This problem has been studied for trees, multi-dimensional grids [4], Petersen graphs [8], torus networks [9], Benes networks [7], honeycomb networks [10], enhanced hypercubes [11], circulant networks [12], circulant and Harary graphs [13].

The metric dimension concepts have some applications in chemistry for representing chemical components, the navigation of robots in networks [4], image processing and pattern recognition. Interconnection networks are designed for use at different levels within and across computer systems to meet the operational demands of various application areas. In Chapter 18 of Parallel and Distributed Computing Handbook, by Albert Y. Zomaya, Stojmenović [14] suggests a number of open problems for various interconnection networks. One of them is about designing new architectures. He writes: "Designing new architectures remains an area of intensive investigation given that there is no clear winner among existing ones”. Motivated by this statement we aim at defining and constructing new architectures and we begin our search in the field of chemical structures.

In this paper we consider graphs of certain crystal structures and determine the metric dimension and fault tolerant metric dimension.

\section{Crystal Structures}

The physical structure of solid materials of engineering importance depends mainly on the arrangements of the atoms, ions, or molecules that make up the solid and the bonding forces between them. If the atoms or ions of a solid are arranged in a pattern that repeats itself in three dimensions, they form a solid that is said to have a crystal structure and is referred to as a crystalline solid or crystalline material. The atomic arrangement or crystalline structure of a material is important in determining the behavior and properties of a solid material. Examples of crystalline materials are metals, alloys, and some ceramic materials. The unit cell is the smallest structural unit or building block that can describe the crystal structure. Repetition of the unit cell generates the entire crystal. In this paper, we investigate the metric dimension and fault-tolerant metric dimension problems for the graphs bismuth tri-iodide, lead chloride and quartz (crystalline $\mathrm{SiO}_{2}$ ).

\subsection{The Graph of Bismuth Tri-Iodide}

Bismuth tri-iodide (BiI3) is an inorganic compound. It is the product of the reaction of bismuth and iodine, which once was of interest in qualitative inorganic analysis. Layered BiI3 crystal is considered to be a three-layered stacking structure, where bismuth atom planes are sandwiched between iodide atom planes, which form the sequence I-Bi-I planes. The periodic stacking of three layers forms rhombohedral BiI3 crystal with R-3 symmetry [15] [16]. The successive stacking of one I-Bi-I layer forms hexagonal structure with symmetry [17] [18]. A single crystal of BiI3 has been synthesized by Nason and Keller [19]. Figure 1 shows one unit of bismuth tri-iodide.

The graph of a single unit of bismuth tri-iodide contains six 4-cycles of which two are on the top, two are in 


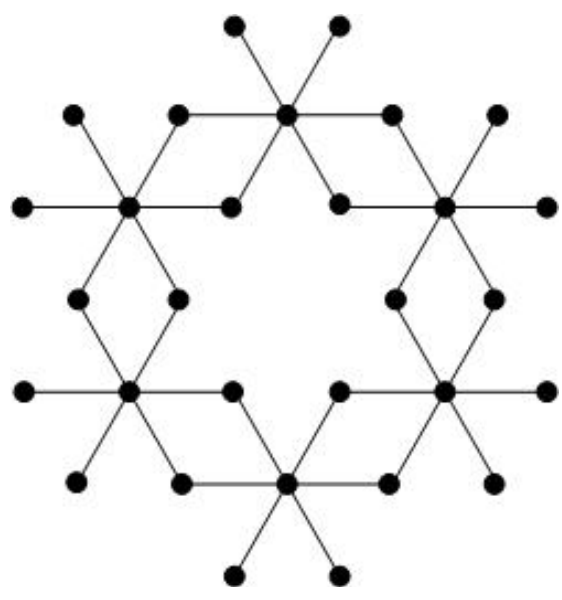

Figure 1. One unit of bismuth tri-iodide.

the middle and two at the bottom. The unit cells of bismuth tri-iodide can be arranged either linearly or in a sheet form. A linear arrangement with $m$ unit cells is called an $m$-bismuth chain; $m n$ unit cells arranged into $m$ rows and $n$ columns is called an $m \times n$ bismuth sheet.

We first obtain a lower bound for the metric dimension of an $m$-bismuth chain.

Lemma 1. Let $G$ be an $m$-bismuth chain. Then $\beta(G) \geq 7 m+5$.

Proof. The two 2-degree vertices of each 4-cycle are equidistant from every vertex of $G$. So one of them must belong to any resolving set. Similarly the two 1-degree vertices incident with a common vertex are equidistant from every vertex of $G$. So one of them must belong to any resolving set. Hence $\beta(G) \geq 7 m+5$.

Our next result realizes the lower bound obtained in Lemma 1.

Theorem 1. Let $G$ be an $m$-bismuth chain. Then $\beta(G) \leq 7 m+5$.

Proof. The cycles in an $m$-bismuth chain are listed as $C_{j}^{T}, C_{k}^{M}$ and $C_{j}^{B}, 1 \leq j \leq 2 m$ and $1 \leq k \leq m+1$. Let $p_{i}, q_{i}, \quad 1 \leq i \leq m+2, \quad x_{j} \in C_{j}^{T}, \quad y_{k} \in C_{k}^{M}$ and $z_{j} \in C_{j}^{B}, 1 \leq j \leq 2 m$ and $1 \leq k \leq m+1$ be the vertices as indicated in Figure 2. We claim that $W=\left\{p_{i}, q_{i}, x_{j}, y_{k}, z_{j}: 1 \leq i \leq m+2,1 \leq j \leq 2 m, 1 \leq k \leq m+1\right\}$ is a resolving set. Let $u, v \in V \backslash W$.

Case 1: $u, v \in C_{j}^{T}$, for some $j, 1 \leq j \leq 2 m$.

If $u$ and $v$ are adjacent, then $x_{j} \in C_{j}^{T}$ resolves them. If $u$ and $v$ are non-adjacent in $C_{j}^{T}$, then they are equidistant from $x_{j}$. In this case either $x_{j-1}$ or $x_{j+1}$ resolves $u$ and $v$. This argument holds for $2 \leq j \leq 2 m-1$. If $j=1$ or $2 m$, then $y_{1} \in C_{1}^{M}$ or $y_{m+1} \in C_{m+1}^{M}$ resolves $u$ and $v$ respectively. The argument is similar if $u, v \in C_{j}^{B}$ or $C_{k}^{M}$, $1 \leq j \leq 2 m$ and $1 \leq k \leq m+1$.

Case 2: $u \in C_{j}^{T}$ and $v \in C_{k}^{M}$.

In this case $u$ and $v$ are resolved by $q_{s}, 1 \leq s \leq m+2$. The argument is similar if $u \in C_{k}^{M}$ and $v \in C_{j}^{B}$ or $u \in C_{j}^{T}$ and $v \in C_{j}^{B}$. In both cases a leaf vertex resolves $u$ and $v$.

Lemma 1 and Theorem 1 solve the metric dimension problem for an $m$-bismuth chain completely. Thus we have the following result.

Theorem 2. Let $G$ be an m-bismuth chain. Then $\beta(G)=7 m+5$.

\section{Fault-Tolerant Metric Dimension}

In the proof of Theorem 2, we constructed a metric basis $W$ consisting of a 2-degree vertex from each 4-cycle and a leaf vertex from each of the pair of leaf incident with a 4-cycle.

Let $W^{*}$ be the set of all 2-degree vertices and all the leaves vertices. Clearly $W^{*}$ is a resolving set being a superset of the constructed resolving set $W$.

It is easy to see that representations of any two vertices $u, v \in V W^{*}$ will differ in at least two distinct places. Hence $W^{*}$ is a fault-tolerant metric basis.

Theorem 3. Let $G$ be an $m$-bismuth chain. Then $\beta^{\prime}(G)=14 m+10$.

We state the results for $m \times n$-bismuth sheet.

Theorem 4. Let $G$ be an $m \times n$-bismuth sheet. Then $\beta(G)=3 m n+4(m+n)+1$.

Theorem 5. Let $G$ be an $m \times n$-bismuth sheet. Then $\beta^{\prime}(G)=6 m n+8(m+n)+2$. 


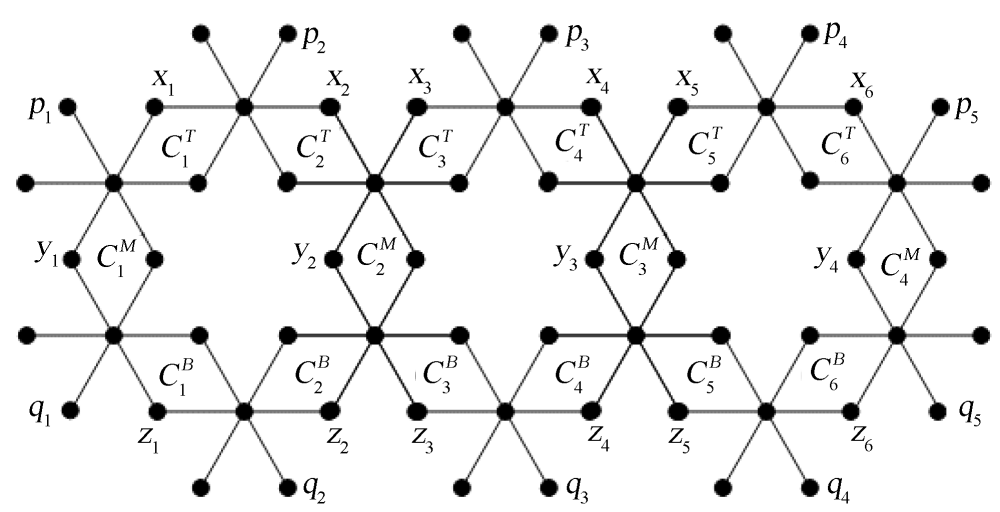

Figure 2. A 3-bismuth chain.

\subsection{The Graph of Lead Chloride}

Lead chloride is a halide crystal which occurs naturally in the form of mineral cotunnite. It is used in the production of infra red transmitting glass and basic chloride of lead known as patteson's white lead, perry, ornamental glass called aurene glass, stained glass. It is also used as an intermediate in refining bismuth (Bi) ore, it is used in the synthesis of organometallic, lead titanate and barium titanate. The structure of lead chloride is orthorhombic dipyramidal.

The graph of a single unit of lead chloride is obtained from that of bismuth tri-iodide by joining just one 2-degree vertex of each of the 4-cycles to a new vertex.

As in the case of bismuth tri-iodide, chains and sheets of lead chloride are defined.

Lemma 2. Let $G$ be an m-lead chloride chain. Then $\beta(G) \geq 2 m+4$.

Proof. Every pair of 1-degree vertices incident with a common vertex are equidistant from every vertex of the graph. Hence at least one vertex must belong to any resolving set. Hence $\beta(G) \geq 2 m+4$.

We adopt a labeling scheme in which vertices are listed level wise (levels 1 to 7$)$ from top to bottom. The notation $l_{i}^{k}$ will depict the ith vertex in level $k$.

Theorem 6. Let $G$ be an m-lead chloride chain. Then $\beta(G) \leq 2 m+4$.

Proof. Let $p_{i}, q_{i}, 1 \leq i \leq m+2$, be the 1-degree vertices as shown in Figure 3 . We claim that $W=\left\{p_{i}, q_{i}: 1 \leq i \leq m+2\right\}$ is a resolving set. Let $u, v \in V W$.

Case 1: $u, v$ belong to the same level.

In view of the symmetry of the graph, it is enough to consider vertices in levels 1 to 4 . Any two vertices on level 1 are resolved by either $p_{i}=l_{i}^{1}$ or $p_{j}=l_{j}^{1}$. Now we consider any two vertices on level 2 . Without loss of generality let us assume that $u=l_{i}^{2}, 2 \leq i \leq 3 m$ and $v=l_{i+j}^{2}, 1 \leq j \leq 3 m+1$. In this case either $p_{1}=l_{1}^{2}$ or $p_{m+2}=l_{3 m+2}^{2}$ resolves $u$ and $v$. In fact if $i=3 s-1,1 \leq s \leq m$, then $d\left(u, l_{1}^{2}\right)=(4 i-2) / 3$ and $d\left(v, l_{1}^{2}\right)=d(u, v)+d\left(u, l_{1}^{2}\right)$. Similar argument will hold if $i=3 s$ or $i=3 s+1$. The argument is similar for any two vertices in the level 3 except for the pairs of vertices $\left(l_{1}^{3}, l_{3}^{3}\right)$ and $\left(l_{3 m+1}^{3}, l_{3 m+3}^{3}\right)$. These pairs are equidistant, respectively, from $l_{1}^{2}$ and $l_{3 m+2}^{2}$. The pair $\left(l_{1}^{3}, l_{3}^{3}\right)$ is resolved by $l_{3 m+2}^{2}$ and the pair $\left(l_{3 m+1}^{3}, l_{3 m+3}^{3}\right)$ is resolved by $l_{1}^{2}$. The similar argument holds for the level 4 .

Case 2: $u$, $v$ belong to different levels.

Different level vertices are resolved by any $p_{i}$ or $q_{j}, 1 \leq i, j \leq m$.

Lemma 2 and Theorem 6 solve the metric dimension problem for an $m$-lead chloride chain completely. Thus we have the following result.

Theorem 7. Let $G$ be an m-lead chloride chain. Then $\beta(G)=2 m+4$.

Theorem 8. Let $G$ be an m-lead chloride chain. Then $\beta^{\prime}(G)=4 m+8$.

\subsection{The Graph of Quartz (Crystalline $\mathrm{SiO}_{2}$ )}

Quartz is a crystalline form of silicon dioxide with formula $\mathrm{SiO}_{2}$. A quartz network can be built in various ways. The quartz network of dimension 1 is a 12-cycle. The quartz network of dimension $n$ consists $n^{2}$ number of 12-cycles and these 12-cycles are arranged in the shape of a rhombus. Figure 4 depicts a quartz network of 


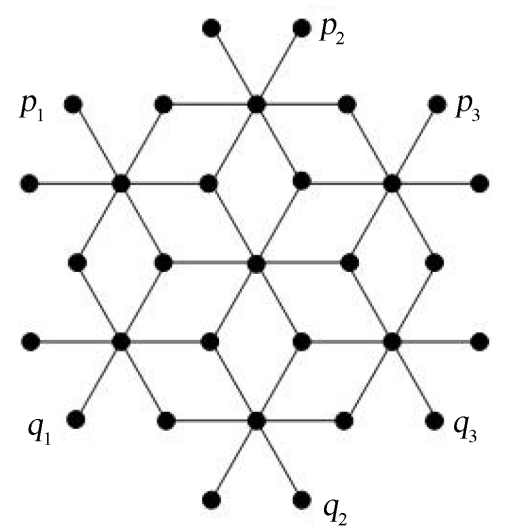

Figure 3. One unit of lead chloride.

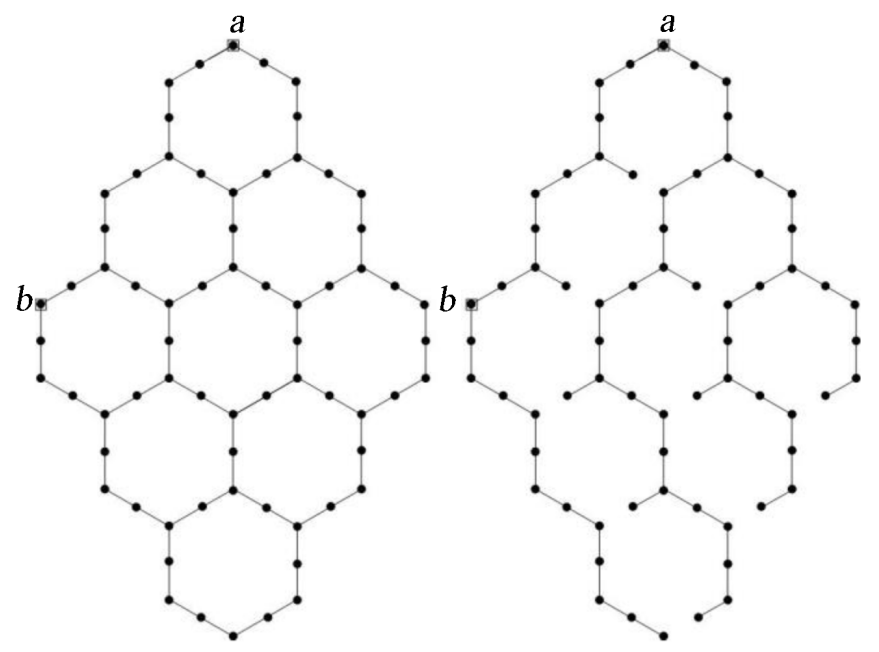

Figure 4. A quartz network of dimension 3 with basis $=\{a, b\}$.

dimension 3.

Theorem 2.11. Let $G$ be the graph of quartz. Then $\beta(G)=2$.

Proof. It is easy to see that $\{a, b\}$ is a resolving set. This follows by using the underlying binarytree structure rooted at $a$. Vertices at different levels are resolved by the root and vertices at the same level are resolved by $b$.

Theorem 2.12. Let $G$ be the graph of quartz. Then $\beta^{\prime}(G)=4$.

\section{Conclusion}

In this paper we have solved the metric dimension and fault-tolerant metric dimension problems for the graphs of certain crystal structures. This problem is under investigation for certain nanostructures.

\section{References}

[1] Slater. P.J. (1975) Leaves of Trees. Proceeding of the 6th Southeastern Conference on Combinatorics, Graph Theory, and Computing, Congressus Numerantium, 14, 549-559.

[2] Harary, F. and Melter, R.A. (1976) On the Metric Dimension of a Graph. ArsCombinatoria, 2, 191-195.

[3] Melter, R.A. and Tomescu, I. (1984) Metric Bases in Digital Geometry. Computer Vision, Graphics, and Image Processing, 25, 113-121. http://dx.doi.org/10.1016/0734-189X(84)90051-3

[4] Khuller, S., Ragavachari, B. and Rosenfeld, A. (1996) Landmarks in Graphs. Discrete Applied Mathematics, 70, 217229. http://dx.doi.org/10.1016/0166-218X(95)00106-2

[5] Hernando, C., Mora, M., Pelayo, I.M., Seara, C., Cáceres, J. and Puertas, M.L. (2005) On the Metric Dimension of 
Some Families of Graphs. Electronic Notes in Discrete Mathematics, 22, 129-133. http://dx.doi.org/10.1016/j.endm.2005.06.023

[6] Garey, M.R. and Johnson, D.S. (1979) Computers and Intractability: A Guide to the Theory of NP-Completeness. Freeman, New York.

[7] Manuel, P., Abd-El-Barr, M.I., Rajasingh, I. and Rajan, B. (2008) An Efficient Representation of Benes Networks and its Applications. Journal of Discrete Algorithms, 6, 11-19. http://dx.doi.org/10.1016/j.jda.2006.08.003

[8] Rajan, B., Rajasingh, I., Cynthia, J.A. and Manuel, P. (2003) On Minimum Metric Dimension. Proceedings of the Indonesia-Japan Conference on Combinatorial Geometry and Graph Theory, Bandung, September 2003, 13-16.

[9] Manuel, P., Rajan, B., Rajasingh, I. and Chris Monica, M. (2006) Landmarks in Torus Networks. Journal of Discrete Mathematical Sciences and Cryptography, 9, 263-271. http://dx.doi.org/10.1080/09720529.2006.10698077

[10] Manuel, P., Rajan, B., Rajasingh, I. and Chris Monica, M. (2008) On Minimum Metric Dimension of Honeycomb Networks. Journal of Discrete Algorithms, 6, 20-27. http://dx.doi.org/10.1016/j.jda.2006.09.002

[11] Rajan, B., Rajasingh, I., Chris Monica, M. and Manuel, P. (2008) Metric Dimension of Enhanced Hypercube Networks. The Journal of Combinatorial Mathematics and Combinatorial Computating, 67, 5-15.

[12] Rajan, B., Rajasingh, I., Venugopal, P. and Chris Monica, M. (2014) Minimum Metric Dimension of Illiac Networks. ArsCombinatoria, CXVII, 95-103.

[13] Grigorious, C., Manuel, P., Miller, M., Rajan, B. and Stephen, S. (2014) On the Metric Dimension of Circulant and Harary Graphs. Applied Mathematics and Computation, 248, 47-54. http://dx.doi.org/10.1016/j.amc.2014.09.045

[14] Stojmenovic, I. (1996) Direct Interconnection Networks. In: Zomaya, A.Y., Ed., Parallel and Distributed Computing Handbook, McGraw-Hill, 537-567.

[15] Watanabe, K., Karasawa, T., Komatsu, T. and Kaifu, Y. (1986) Optical Properties of Extrinsic Two-Dimensional Excitons in BiI3 Single Crystals. Journal of the Physical Society of Japan, 55, 897. http://dx.doi.org/10.1143/JPSJ.55.897

[16] Wyckoff, R.W.G. (1964) Crystal Structures, Vol. 2. 2nd Edition, John Wiley \& Sons, Inc., New York, London, Sydney.

[17] Schlüter, M., Cohen, M.L., Kohn, S.E. and Fong, C.Y. (1976) Electronic Structure of BiI $\mathrm{I}_{3}$ Physica Status Solidi (B), 78, 737. http://dx.doi.org/10.1002/pssb.2220780235

[18] Yorikawa, H. and Muramatsu, S. (2008) Theoretical Study of Crystal and Electronic Structures of BiI 3 . Journal of Physics: Condensed Matter, 20, 325220.

[19] Nason, D. and Keller, L. (1995) The Growth and Crystallography of Bismuth Tri-Iodide Crystals Grown by Vapor Transport. Journal of Crystal Growth, 156, 221. http://dx.doi.org/10.1016/0022-0248(95)00291-X 